\title{
Cardiac surgery in adults performed at children's hospitals: Trends and outcomes
}

\author{
William T. Mahle, MD, ${ }^{a}$ Paul M. Kirshbom, MD, ${ }^{b}$ Kirk R. Kanter, MD, ${ }^{b}$ and Brian M. Kogon, MD $^{\mathrm{b}}$
}

From the Children's Healthcare of Atlanta and the Department of Pediatrics, ${ }^{\mathrm{a}}$ and the Emory Clinic, Department of Cardiothoracic Surgery, ${ }^{\text {b }}$ Emory University School of Medicine, Atlanta, Ga.

Received for publication Dec 12, 2007; revisions received Jan 23, 2008; accepted for publication April 13, 2008.

Address for reprints: William T. Mahle, MD, Children's Healthcare of Atlanta, Emory University School of Medicine, 52 Executive Park South, Suite 52, Atlanta, GA 30329 (E-mail: mahlew@kidsheart.com).

J Thorac Cardiovasc Surg 2008;136:307-11 $0022-5223 / \$ 34.00$

Copyright $\odot 2008$ by The American Association for Thoracic Surgery

doi:10.1016/j.jtcvs.2008.04.020
Objective: The number of adults with congenital heart disease who require cardiac surgery is projected to increase dramatically. Controversy exists as to whether such procedures should be performed in pediatric centers, which generally have the greatest experience with operations for congenital heart disease. We sought to report the outcomes for cardiac surgery performed in adults ( $\geq 21$ years of age) at children's hospitals and determine how these practices varied among institutions.

Methods: Data from July 2005 to June 2007 from the Child Health Corporation of America, a consortium of 37 free-standing children's hospitals, were analyzed to determine the institutional volume, type of cardiac procedure, outcome, and hospital charges. Individual institutional variables were analyzed to determine which factors might be associated with the practice of performing adult cardiac surgery in children's hospitals.

Results: During the study period, there were 719 admissions for cardiac surgery in adults at Child Health Corporation of America institutions. The median age at the time of operation was 26 years (range, $21-86$ years). The most common surgical procedures were implantation or revision of a pacemaker or defibrillator $(\mathrm{n}=207$ [29.2\%]), pulmonary valve replacement $(\mathrm{n}=119$ [16.8\%]), aortic valve replacement $(\mathrm{n}=59[8.3 \%])$, and Fontan revision $(\mathrm{n}=37$ [5.2\%]). The median hospital length of stay was 6 days (range, $1-175$ days). The hospital mortality was 1.9\%. Comorbid conditions likely to require other subspecialty care were present in more than $30 \%$ of patients. Among the Child Health Corporation of America centers, adult operations as a proportion of overall cardiac operations varied from $0 \%$ to $10.9 \%$. There was no relationship between overall cardiac surgical volume and proportion of adult cases performed in Child Health Corporation of America centers.

Conclusions: A significant number of adult cardiac surgical procedures are being performed at children's hospitals with excellent results. The majority of procedures are not related to complex shunt lesions but rather pacemaker/defibrillator implantation and semilunar valve surgery. Whether adult patients with congenital heart disease should continue to undergo most cardiac surgery in children's hospitals is worthy of discussion.

$\mathrm{T}$ There will be an estimated 400,000 adults with moderate or complex congenital heart disease (CHD) in the United States by 2010. ${ }^{1}$ A significant number of these adult patients will require additional cardiac surgical procedures for residual lesions, such as pulmonary valve regurgitation, atrioventicular valve regurgitation, outflow tract obstruction, or arrhythmias. ${ }^{2,3}$ Such operations have traditionally been performed by congenital heart surgeons, the great majority of whom have focused on pediatric patients. ${ }^{4}$ Because of the increasing number of adult patients with CHD, there has been interest in creating regional adult CHD centers. ${ }^{1}$ In these centers patients could receive medical and surgical care for CHD and access to relevant subspecialists. ${ }^{5,6}$ Given that adults can have comorbid conditions that are uncommon in children, such as deep venous thrombosis, atherosclerotic coronary artery 


\section{Abbreviations and Acronyms \\ $\mathrm{CHCA}=$ Child Health Corporation of America \\ $\mathrm{CHD}=$ congenital heart disease}

disease, or peripheral vascular disease and emphysema, it might be that adult patients with CHD are best served in adult hospitals with specialized congenital heart cardiologists and surgeons. An alternative approach is to perform adult CHD surgery in children's hospitals that are familiar with CHD physiology.

In the present study we sought to determine the surgical volume and outcomes for CHD procedures performed in adults at children's hospitals. In addition, we endeavored to determine whether there were geographic or institutional factors that we associated with the relative volume of adult CHD procedures performed in children's hospitals.

\section{Materials and Methods}

With the approval of the Institutional Review Board of Emory University School of Medicine, we analyzed data from the Pediatric Health Information System, which is a large inpatient administrative database of 37 participating children's hospitals in the Child Health Corporation of America (CHCA), a children's hospital consortium. Detailed information on demographics, diagnoses, procedures, medications, and outcomes is available for every inpatient (all patient-level data are deidentified). Data are subjected to a number of quality checks. Data are accepted into the database when classified errors less frequently than a criterion threshold of less than $2 \%$ of a hospital's data. The present study was limited to July 1, 2005, to June 30, 2007. Subjects were identified by searching principal diagnosis and procedures codes of the Pediatric Health Information System Cardiac Surgery service line, a group of APR-Diagnostic Related Groups. Subsequently, diagnoses and procedures were determined by using International Classification of Diseases-ninth revision codes. Relevant patient variables available in the database include demographic data, as well as duration of hospital length of stay, survival to hospital discharge, and total hospital charges. Race (white, black, Asian, and "other") and payer status (Medicaid, Blue Cross/ other insurance, other government program, or "other") were collected.

To examine the factors specific to each children's hospital, we also analyzed the total number of hospital discharges associated with CHD. We also sought to determine whether the presence of an adjoining or nearby adult academic medical center might influence the approach to performing adult cardiac surgery in a children's hospital. The CHCA centers were categorized as (1) directly attached or sharing the same campus or (2) not affiliated with an academic medical center with a moderate adult cardiac surgical volume. In addition, we examined whether children's hospitals with high cardiac volume were more likely to perform cardiac surgery in adults.

For continuous variables, the Mann-Whitney $U$ test was used. For categorical variables, Fisher's exact tests were performed.

\section{Results}

During the 24-month study period, there were a total of 719 total surgical discharges. The median age at the time of operation was 26 years (range, $21-86$ years). Subjects 40 years of age or older comprised $13.3 \%$ of the population. The most common principal diagnosis was tetralogy of Fallot, followed by arrhythmia (Table 1). The most commonly performed procedures were implantation or revision of a pacemaker/ defibrillator, replacement of a pulmonary valve, and replacement or repair of a pulmonary valve (Table 2 ).

The median number of cardiac operations performed per children's hospital in the CHCA was 7. The highest-volume center had 1162 total cardiac surgical discharges, of which 103 were of adults. Mean adjusted hospital charge per patient was $\$ 126,258$. The procedures with the highest mean charges were heart transplantation, followed by the Fontan procedure, which had a mean adjusted hospital charge of $\$ 818,175$ and $\$ 291,597$, respectively. There was no difference in the mean charge per case based on overall surgical volume $(P=.36)$.

The proportion of total cardiac operations performed in adults 21 years and older varied considerably among the children's hospitals. Some children's hospitals performed no cardiac operations in adults, whereas in one CHCA center adult cardiac operations accounted for $10.9 \%$ of the entire cardiac surgical volume. Among the 5 programs with the highest overall cardiac surgical volume, there was considerable variability. For 2 of the high-volume centers, adult cardiac operations accounted for less than $0.2 \%$ of the overall cardiac surgical procedures. Among the remaining 3 high-volume centers, adult procedures accounted for between $3.3 \%$ and $7.4 \%$ of overall surgical case load. Location on the campus of an academic medical center with a moderate-volume adult

\section{TABLE 1. Most common primary cardiac diagnoses}

\begin{tabular}{lc}
\hline & No. (\%) \\
\hline Tetralogy of Fallot & $108(15.0)$ \\
Cardiac arrhythmia & $84(11.7)$ \\
Malfunction of pacemaker & $48(6.7)$ \\
Malfunction AICD & $24(3.3)$ \\
Secundum ASD & $36(5.0)$ \\
Ventricular septal defect & $28(3.9)$ \\
Functional single ventricle & $40(5.4)$ \\
Double outlet right ventricle & $15(2.1)$ \\
Aortic insufficiency & $22(3.1)$ \\
Malfunction prosthetic valve & $18(2.5)$ \\
Transposition of the great arteries & $14(1.9)$ \\
Coarctation of the aorta & $17(2.4)$ \\
Atrioventricular septal defect & $16(2.2)$ \\
Ebstein anomaly & $10(1.4)$ \\
Other & $239(33.2 \%)$
\end{tabular}

$A I C D$, Automatic implantable cardioverter defibrillator; $A S D$, atrial septal defect. 
TABLE 2. Most common principal cardiac surgical procedures and hospital mortality

\begin{tabular}{|c|c|}
\hline & No. $(\%)$ \\
\hline Pulmonary valve replacement & $119(16.6)$ \\
\hline Revision of pacemaker/defibrillator & $75(10.4)$ \\
\hline Defibrillator implantation & $68(9.5)$ \\
\hline Pacemaker implantation & $64(8.9)$ \\
\hline Aortic valve replacement & $59(8.2)$ \\
\hline Fontan procedure revision & $37(5.1)$ \\
\hline Atrioventricular valve repair & $35(4.9)$ \\
\hline ASD closure & $28(3.9)$ \\
\hline Atrioventricular valve replacement & $27(3.8)$ \\
\hline RV to PA conduit replacement & $18(2.5)$ \\
\hline Aortic valve repair & $13(1.8)$ \\
\hline Heart transplantation & $12(1.7)$ \\
\hline Coarctation of the aorta & $10(1.4)$ \\
\hline VSD closure & $9(1.3)$ \\
\hline Other procedures & $145(20.1)$ \\
\hline
\end{tabular}

$A S D$, Atrial septal defect; $R V$, right ventricle; $P A$, pulmonary artery; $V S D$, ventricular septal defect.

cardiac surgery program did not appear to influence the practice of adult cardiac surgery at children's hospitals (Table 3). Those children's hospitals that shared a campus with an adult heart program performed a median of $3.7 \%$ of all their cases in adults compared with $1.9 \%$ of overall cardiac volume for the CHCA hospitals not sharing a campus with an adult facility $(P=.23)$.

\section{TABLE 3. Hospital characteristics}

\begin{tabular}{lc}
\hline & Entire cohort $(\mathbf{n}=\mathbf{3 7})$ \\
\hline Adult academic medical center* & \\
Directly attached & $12(32.4 \%)$ \\
Same campus & $8(21.6 \%)$ \\
Different campus & $10(27.0 \%)$ \\
No affiliation & $7(18.9 \%)$ \\
Mean no. of total CHD surgical & \\
discharges per institution & \\
$>1000$ & 1 \\
$501-1000$ & $4(10.9 \%)$ \\
$401-500$ & $2(5.4 \%)$ \\
$301-400$ & $9(24.3 \%)$ \\
$201-300$ & $13(35.1 \%)$ \\
$<200$ & $8(21.6 \%)$ \\
Mean no. of adult CHD surgical & \\
discharges per institution & \\
$>100$ & $1(2.7 \%)$ \\
$51-100$ & $1(2.7 \%)$ \\
$31-50$ & $4(10.8 \%)$ \\
$21-30$ & $5(13.5 \%)$ \\
$11-20$ & $13(35.1 \%)$ \\
$0-10$ & $13(35.1 \%)$ \\
\hline
\end{tabular}

$C H D$, Congenital heart disease. ${ }^{*}$ Adult cardiac surgical center with at least 200 cardiac procedures per year.
The median hospital length of stay was 6 days (range, 1-175 days). There were 14 hospital deaths, yielding a hospital mortality of $1.9 \%$. Hemodialysis was performed in 12 patients. Extracorporeal membrane oxygenation was used in 8 patients.

Associated medical conditions, complications, or both were common in this patient population (Table 4). Importantly, psychiatric conditions, endocrine abnormalities, and hematologic disorders were reported in more than $15 \%$ of subjects.

\section{Discussion}

The number of adults with CHD is projected to increase markedly in the coming decades, and adult congenital cardiac surgery is likely to become increasingly specialized. Although some have proposed that these procedures be performed in regional adult congenital centers, the present study demonstrates that many adult CHD surgical procedures continue to be performed in children's hospitals. The outcome for adult CHD surgery performed at children's hospitals is quite favorable, with overall mortality of less than $2 \%$.

The great majority of inpatient cardiac care in children is focused on CHD. Conversely, the adult cardiologist has traditionally had little or any exposure to CHD during training. As such, when patients with CHD reach adulthood, pediatric cardiologists often continue to provide care, and inpatient surgical procedures are often performed in a setting in which specialists are familiar with CHD. As these patients transition to adulthood, it is unclear whether they should undergo

TABLE 4. Associated medical conditions among adults $(n=719)$ at children's hospitals

\begin{tabular}{lr}
\hline & No. (\%) \\
\hline Renal & \\
Acute renal failure & $27(3.8)$ \\
Chronic renal failure & $7(1.0)$ \\
Gastrointestinal & \\
Cirrhosis of liver & $8(1.1)$ \\
Acute pancreatitis & $4(0.6)$ \\
Viral hepatitis & $7(1.0)$ \\
Psychiatric & \\
Depression & $19(2.6)$ \\
Anxiety state/panic disorder & $24(3.3)$ \\
Manic-depression & $3(0.4)$ \\
Substance abuse & $21(2.9)$ \\
Hematologic & \\
Blood disorder other than anemia & $46(6.5)$ \\
Endocrinologic & \\
Hypothyroidism & $48(6.4)$ \\
Diabetes & $18(2.5)$ \\
Gout & $6(0.8)$ \\
Obstructive sleep apnea & $14(1.9)$ \\
Alzheimer's dementia & $3(0.4)$ \\
\hline &
\end{tabular}


cardiac surgical procedures in a children's hospital or in an adult facility with likely a smaller volume of CHD cases. Children's hospitals were initially developed to provide specialized care to children not routinely available in hospitals devoted to adults. Such specialization of services has undoubtedly contributed to the remarkable improvement in infants with CHD. Hospital mortality for all forms of CHD operations in children is now less than $2 \%$.

One argument that has been proposed is that adult patients with CHD should continue to undergo surgical procedures at pediatric facilities because these centers have personnel familiar with the physiology and complications unique to CHD such as might occur in those with a functional single ventricle. A recent multicenter analysis from Europe suggested that the majority of adult CHD surgical cases were being performed in pediatric centers. ${ }^{7}$ However, the present study suggests that the great majority of cardiac operations performed in adults with CHD are related to valvar pathology that is not dissimilar to valve operations performed in adults with acquired heart disease. Implantation of pacemakers or defibrillators, replacement of pulmonary valves, aortic valve repair or replacement, atrioventricular valve repair or replacement, and operations for simple shunt lesions accounted for more than $70 \%$ of all the adult CHD procedures performed in children's hospitals. Although the technical expertise of a surgeon trained in congenital heart lesions would seem crucial, the postoperative physiology for many of the subjects would not require management of complex intracardiac shunts. A recent multicenter report from Europe that included 2012 adults undergoing CHD also demonstrated palliative operations or complex procedures, such as the Fontan operation and operations for Ebstein's anomaly, and complete repair of tetralogy of Fallot accounted for only $7 \%$ of the CHD operations performed in adults. ${ }^{7}$ Given the rarity of cyanotic heart disease or complex postoperative shunts, the argument that caregivers be familiar with unique CHD physiology would seem less compelling.

The overall hospital mortality for adult CHD operations in this series was quite low at $1.6 \%$. This is in agreement with the results of 2 other published series of operations in adults with $\mathrm{CHD}^{7,8}$ As the volume of adult CHD operations increases, it will be important to develop benchmarks and risk-stratification models. Recently, investigators have developed risk-stratification models for CHD operations, including the risk-adjusted classification for congenital heart surgery model. ${ }^{9}$ This model was derived from congenital cardiac surgical data in patients less than 18 years of age. Therefore the extent to which this model might be relevant to the adult patient with CHD is not known. Similarly, the Aristotle model has also been designed to account for differences in CHD surgical mortality in children and adolescents. ${ }^{9}$ In the Society of Thoracic Surgeons Database more than 57\% of CHD operations were performed in children less than 12 months of age. Importantly, cardiac procedures performed in children older than 12 months resulted in hospital mortality of $1.2 \%$. Therefore in evaluating the outcome of adult CHD operations, whether performed in freestanding children's hospitals or adult medical centers, hospital mortality might not be the most appropriate measure for benchmarking. Other factors, such as length of stay, hospital costs, or complications, might be more appropriate metrics. Hopefully, in the coming years models can be developed to measure quality outcomes in adult CHD operations. Such a model might be able to address the relative advantages or disadvantages of performing CHD operations in children's hospitals versus adult centers.

The present study demonstrates that the approach to the management of adult CHD operations varies significantly throughout the United States. Clearly, some centers or regions have moved toward a strategy of transitioning patients to adult centers for cardiac surgery. For just more than one third of CHCA centers, adult patients with CHD account for less than $4 \%$ of the overall CHD volume. On the other hand, in $13 \%$ of CHCA hospitals, adult patients account for more than $10 \%$ of the overall CHD surgical discharges. These differences are dramatic and might be dictated by a variety of factors. There did not appear to be any significant relationship between overall CHD surgical volume and the likelihood that more than $10 \%$ of all CHD cases were performed in adults. In addition, having an affiliated adult academic medical center on the same campus did not seem to influence the likelihood that a children's hospital would perform a relatively high number of adult CHD operations.

One of the arguments that would support the idea that cardiac surgery in adults with CHD is best performed in adult centers relates the additional subspecialty care that is required. Comorbid conditions were relatively common in this population. Some of the conditions reported are extremely rare in the pediatric population and hence might present a challenge to pediatric providers. Among the conditions reported in the study population were gout, Alzheimer's dementia, and emphysema. A number of pediatric subspecialists, such as child neurologists and psychiatrists, generally have had some training in adult care. In the United States other subspecialists, such as pediatric endocrinologists, gastroenterologists, or nephrologists, generally have had very little, if any, exposure to adult care, and management of end-organ complications in the adult with CHD can present a significant challenge.

In summary, a significant number of adults with CHD undergo cardiac surgery at children's hospitals in the United States. The majority of surgical procedures do not involve complex intracardiac techniques. The outcome for adult cardiac surgery performed in children's hospitals is excellent, with hospital survival of more than $98 \%$.

We thank Mr Chuck Cochran and the CHCA for their support with this project. 


\section{References}

1. Williams RG, Pearson GD, Barst RJ, Child JS, del NP, Gersony WM, et al. Report of the National Heart, Lung, and Blood Institute Working Group on research in adult congenital heart disease. J Am Coll Cardiol. 2006;47:701-7.

2. Chatzis AC, Giannopoulos NM, Tsoutsinos AI, Chryssostomidis G, Panagiotou C, Zavaropoulos P, et al. Successful surgical correction of congenital heart disease in adults: seven years' experience. Hellenic $J$ Cardiol. 2005;46:128-34.

3. Klcovansky J, Sondergaard L, Helvind M, Andersen HO. Cardiac surgery in grown-up congenital heart patients. Will the surgical workload increase? Interact Cardiovasc Thorac Surg. 2008;7:89.

4. Jacobs ML, Mavroudis C, Jacobs JP, Tchervenkov CI, Pelletier GJ. Report of the 2005 STS Congenital Heart Surgery Practice and Manpower Survey. Ann Thorac Surg. 2006;82:1152-5.
5. Daebritz SH. Update in adult congenital cardiac surgery. Pediatr Cardiol. 2007;28:96-104.

6. Dearani JA, Connolly HM, Martinez R, Fontanet H, Webb GD. Caring for adults with congenital cardiac disease: successes and challenges for 2007 and beyond. Cardiol Young. 2007;17(suppl 2): 87-96.

7. Vida VL, Berggren H, Brawn WJ, Daenen W, Di CD, Di DR, et al. Risk of surgery for congenital heart disease in the adult: a multicentered European study. Ann Thorac Surg. 2007;83:161-8.

8. Mott AR, Fraser CD Jr, McKenzie ED, Bezold LI, Andropoulos DB, Reul GJ, et al. Perioperative care of the adult with congenital heart disease in a free-standing tertiary pediatric facility. Pediatr Cardiol. 2002;23: 624-30.

9. Jenkins KJ. Risk adjustment for congenital heart surgery: the RACHS-1 method. Semin Thorac Cardiovasc Surg Pediatr Card Surg Annu. 2004; $7: 180-4$. 